\title{
Mutación del gen K-ras en el cáncer de la vesícula biliar
}

\author{
Juan Carlos Roa S', Iván Roa E ${ }^{1}$, Xabier de Aretxabala U 2 , \\ Angélica Melo $A^{1}$, G aspar Faría $0^{2}$, 0 scar Tapia $E^{1}$. \\ K-ras gene mutation \\ in gallbladder carcinoma
}

Background: Different K-ras mutation frequencies in gallbladder cancer have been reported. Aim: To study the frequency of K-ras gene mutations in advanced gallbladder carcinoma not associated to anomalous junction of pancreatic-biliary duct (AJPBD). Material and methods: 33 formalin fixed paraffin embedded samples of gallbladder carcinoma (30 women, age range 32-86 years) were selected. Pancreatic cancer tissue with Kras mutations was used as control. DNA was extracted from the histological section by mean of microdissection and K-ras mutations in codon 12 were detected by polymerase chain reaction and restriction fragment length polymorphism (RFLP), using previously reported technique. Results: Most cases were poorly differentiated adenocarcinomas. K-ras mutation was detected in 10 cases (30\%) samples. No differences in K-ras mutation frequency between subserous and serous tumors were detected and no relation between histological features and the mutation was observed. Conclusions: K-ras mutation in codon 12 is present in $30 \%$ in our advanced gallbladder carcinomas. The study of K-ras mutation in preneoplastic lesions and early carcinomas will be important to determine the role of this gene in the gallbladder carcinogenesis in Chile (Rev Méd Chile 2004; 132: 955-60).

(Key Words: Gallbladder neoplasms; Genes, ras; K-ras gene)

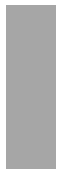

Recibido el 18 de diciembre, 2003. Aceptado en versión corregida el 1 de junio, 2004.

Trabajo financiado por Proyecto de la Dirección de Investigación Universidad de la Frontera 140305.

Departamentos de Anatomía Patológica ${ }^{1}$ y Cirugía ${ }^{2}$, Facultad de Medicina, Universidad de La Frontera.

L a familia de genes ras (H-ras, N-ras y K-ras) es uno de los grupos de oncogenes más frecuentemente alterados en las neoplasias humanas ${ }^{24,25}$. Las proteínas codificadas por estos genes se ensamblan entre sí, conformando una estructura proteica con un peso de $21 \mathrm{Kd}$ que le otorga el

Correspondencia a: Dr. Iván Roa E. Casilla 54-D. Facultad de Medicina, Universidad de La Frontera, Temuco, Chile. E mail: iroa@ufro.cl nombre (p21). Poseen actividad GTP-asa, participan en la vía de transducción de señales de crecimiento y diferenciación celular ${ }^{25,26}$. Las proteínas p21 mutadas se activan constitutivamente y estimulan el crecimiento y la diferenciación de manera autónoma. En alrededor del $80 \%$ de las mutaciones observadas en el gen K-ras se localizan en el codón 12 y, en menor frecuencia, en los codones 13, 60, 61. La mutación del codón 12 ocurre en el segundo nucleótido y corresponden predominantemente a transiciones (guanina-ade- 
nina) con sustitución de ácido aspártico por glicina $^{27,28}$.

En el cáncer de la vesícula biliar en pacientes sin anomalías en la unión pancreático-biliar,29-31, se han comunicado porcentajes de mutación del gen K-ras que fluctúan entre el 0\% y 60\%5,9,10,30,32. Las diferencias mencionadas en los porcentajes de activación de K-ras sobrepasan lo que pudiese esperarse producto de las variaciones propias de los tumores ${ }^{10}$, pudiendo ser atribuidas al uso de diferentes técnicas empleadas más que a las variaciones de las poblaciones bajo estudio. Se ha utilizado por ejemplo, detección y tipificación de mutaciones en el gen K-ras mediante PCR combinada con el uso de enzimas de restricción (RFLP) ${ }^{30}$, como también mediante polimorfismo conformacional de cadena simple (SSCP) ${ }^{33}$ o electroforesis en gel con gradiente denaturante (DGGE) ${ }^{34}$ seguido de secuenciación ${ }^{35}$. Cada uno de estos métodos con distinta sensibilidad. En el espectro de los resultados, hay autores que no han demostrado mutación de este gen, otros, en cambio, muestran cifras de mutación de entre $50 \%$ y $60 \%$ en los cánceres vesiculares y le asignan una importante participación en la carcinogénesis vesicular ${ }^{16,31,36}$.

El objetivo de este trabajo es determinar la presencia de mutaciones en el codón 12 del gen K-ras en carcinomas avanzados de la vesícula biliar mediante reacción de polimerasa en cadena (PCR) en seminido y RFLP (Restriction fragment length polymorphism).

\section{Material y MÉTOdo}

Casos. Se incluyeron 33 casos de cáncer avanzado de la vesícula biliar de la Unidad de Anatomía
Patológica del Hospital Temuco. En todos los casos, el nivel máximo de infiltración tumoral fue establecido mediante el mapeo completo de la pieza quirúrgica. Como controles se utilizó casos de cáncer de páncreas en los que previamente se había demostrado la mutación del codón 12 del gen K-ras.

Extracción del ADN. Se realizó microdisección manual en 5 cortes histológicos con áreas representativas del tumor. El ADN fue extraído con el Kt Puregene $^{\mathrm{TM}}$ DNA Isolation System (Gentra Systems, USA), de acuerdo a las instrucciones del fabricante. El ADN se almacenó a $-20^{\circ} \mathrm{C}$ hasta su análisis. En todos los casos se realizó amplificación previa del gen ß-globina para verificar su calidad.

Detección de mutaciones del codón 12 del gen Kras. Se utilizó un PCR en seminido y RFLP, con iniciadores previamente reportados en la literatu$\mathrm{ra}^{40}$. Estos iniciadores ( ficar un fragmento del gen K-ras que incluye un sitio de restricción para la enzima MvaI. Las secuencias de los iniciadores y los tamaños de los fragmentos se muestran en la Tabla 1. Tanto los productos PCR (pPCR) de la primera como de la segunda amplificación, fueron digeridos con la enzima MvaI (10 U para $20 \mathrm{ul} \mathrm{pPCR).} \mathrm{El} \mathrm{iniciador}$ $\mathrm{K}$-ras-A tiene una base cambiada con respecto a la secuencia del gen K-ras, esto produce un sitio de restricción para la enzima MvaI cuando el codón 12 es normal. También el iniciador K-ras$\mathrm{B}$ tiene una base cambiada, lo que lo hace susceptible de digestión por la enzima MvaI y de esta manera no interfiere en la $2^{\mathrm{a}}$ amplificación.

Los pPCR de la segunda amplificación fueron corridos en un gel al 10\% de poliacrilamida (1:29),

Tabla 1. Secuencia de los iniciadores K-ras, tamaño de los fragmentos y protocolos de PCR usados

\begin{tabular}{|c|c|c|c|}
\hline Iniciadores & Secuencia 5'-3' & $\begin{array}{l}\text { Tamaño } \\
\text { fragmento }\end{array}$ & Protocolo PCR \\
\hline K-ras-A sense & ACTGAATATAAACTTGTGGTAGTTGGACCT & \multirow[t]{2}{*}{$157 \mathrm{pb}$} & 1a amplificación 35 ciclos, \\
\hline K-ras-B antisense & TCAAAGAATGGTCCTGGACC & & $\begin{array}{l}\mathrm{T}^{\circ} \text { Hibridación } 55^{\circ} \mathrm{C} \mathrm{MgCl} \\
2,5 \mathrm{mM}\end{array}$ \\
\hline K-ras-A sense & ACTGAATATAAACTTGTGGTAGTTGGACCT & \multirow[t]{2}{*}{$129 \mathrm{pb}$} & $2^{\mathrm{a}}$ amplificación 25 ciclos \\
\hline K-Ras-C antisense & GCATATTAAAACAAGATTTAC & & $\begin{array}{l}\mathrm{T}^{\circ} \text { Hibridación } 59^{\circ} \mathrm{C} \mathrm{MgCl} \\
2,5 \mathrm{mM}\end{array}$ \\
\hline
\end{tabular}


Buffer TBE 1X, Glicerol 5\% a 150 volt por $2 \mathrm{~h}$ y posteriormente teñidos con bromuro de etidio.

Controles. Como control positivo, se empleó ADN de carcinomas de páncreas en los que previamente se había demostrado la mutación del K-ras en el codón 12. Como control negativo se usó DNA genómico comercial (Promega) y como control blanco se sustituyó el ADN por agua deionizada.

\section{RESULTADOS}

Las características generales del grupo estudiado se resumen en la Tabla 2. La mayoría de los casos correspondieron a mujeres (90\%) con un promedio de edad de 55,1 años (DS 14,6). La totalidad de los casos correspondieron a adenocarcinomas avanzados (subserosos o serosos) y en su gran mayoría eran tumores con un bajo grado de diferenciación.

En 10 de los 33 casos (30,3\%) se detectó la mutación en el codón 12 del gen K-ras (Figura 1). En los tumores con infiltración de la subserosa se observó una leve mayor frecuencia de mutación respecto de los serosos (Tabla 3), sin embargo, esta diferencia no fue significativa. Tampoco se observó relación entre el grado de diferenciación de los tumores y presencia de la mutación del gen K-ras.

\section{DISCUSIÓN}

Nuestros hallazgos muestran la presencia de mutación del codón 12 del gen K-ras en alrededor
Tabla 2. C aracterísticas generales de los cánceres de vesícula biliar

\begin{tabular}{|lcc|}
\hline & Diagnóstico & $\mathrm{N}$ \\
\hline Tipo histológico & Adenocarcinoma & 33 \\
& Bien & 3 \\
Diferenciación & Moderado & 18 \\
& Poco & 12 \\
Infiltración & Serosa & 16 \\
& Subserosa & 17 \\
Edad (años) & Rango (Prom) & $32-86(55,1)$ \\
Sexo & Hombre & 3 \\
& Mujeres & 30 \\
\hline
\end{tabular}

Tabla 3. M utación gen K-ras (codón 12), grado de infiltración y diferenciación histológica

\begin{tabular}{|ccc|}
\hline Infiltración & Diferenciación & K-ras Mutado \\
\hline Subseroso & Bien & $1 / 2$ \\
& Moderado & $3 / 11$ \\
& Poco & $2 / 4$ \\
Seroso & Bien & $0 / 1$ \\
& Moderado & $2 / 7$ \\
& Poco & $2 / 8$ \\
\hline
\end{tabular}

de un tercio de los carcinomas vesiculares avanzados. Estos resultados demostrarían que este gen estaría activado y jugaría un rol en la carcinogénesis vesicular al igual que en otras neoplasias bilio-

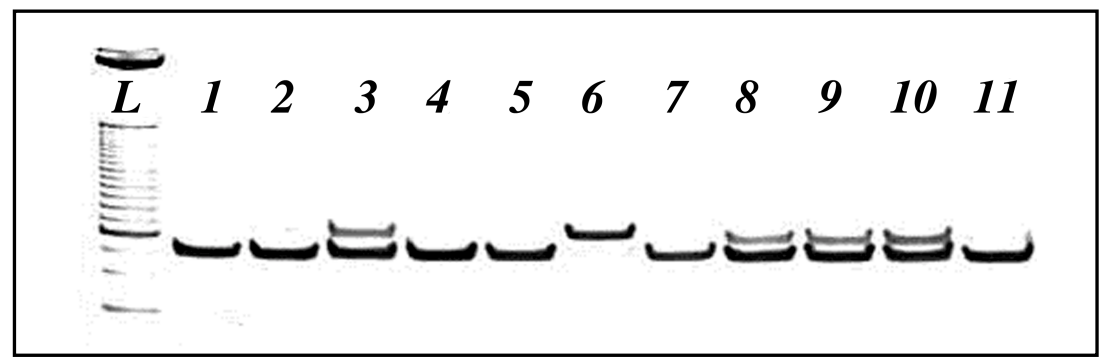

Figura 1. Gel de poliacrilamida teñido con bromuro de etidio. L: marcador 25 bp 1-5 y 7-11. Muestras de cánceres vesiculares, 6: control positivo (cáncer de páncreas). 
pancreáticas ${ }^{8,37}$. Estudios recientes de lesiones displásicas han demostrado que la mutación de este gen sería un evento temprano en la carcinogénesis vesicular ${ }^{16,31,32}$.

Algunos autores han demostrado una baja frecuencia de mutación de este gen en el cáncer vesicular, por lo que le asignan una escasa participación en el desarrollo de esta neoplasia ${ }^{16,38}$, otros en cambio, muestran cifras de mutación que fluctúan entre el 40\%-60\% de los cánceres vesiculares ${ }^{16,30,32}$. Los pacientes portadores de una unión pancreático-biliar anómala, condición frecuente en el oriente, presentan una mayor frecuencia de mutación del gen K-ras que los sujetos sin esta condición, sugiriendo además que el reflujo bilio-pancreático pudiese jugar un rol en la carcinogénesis de este tipo de pacientes ${ }^{35,39}$. Kim y cols, detectaron mutaciones del gen

\section{REFERENCIAS}

1. SERRA I. ¿Ha disminuido la mortalidad por cáncer vesicular en Chile? Rev Méd Chile 2001; 129: 107984.

2. Medina E, KaEMPFFER AM. Mortalidad por cáncer en Chile: consideraciones epidemiológicas. Rev Méd Chile 2001; 129: 1195-202.

3. Nervi F. Cáncer de la vesícula biliar en Chile. Rev Méd Chile 2001; 129: 979-81.

4. Roa I, Melo A, Roa J, Araya J, Viliaseca M, de AREtXabala X. Mutación del gen p53 en el cáncer de la vesícula biliar. Rev Méd Chile 2000; 128: 251-8.

5. Wistuba II, Sugio K, Hung J, Kshimoto Y, Virmani AK, RoA I ET AL. Allele-specific mutations involved in the pathogenesis of endemic gallbladder carcinoma in Chile. Cancer Res 1995; 55: 2511-5.

6. Sanz P, Calvo A, Tobella L, Salazar S, Daher V, CASTILO S ET AL. Chromosome anomaly and flow cytometry in gallbladder adenocarcinoma. Rev Méd Chile 1998; 126: 1301-10.

7. Wistuba II, Albores-SaAvedra J. Genetic abnormalities involved in the pathogenesis of gallbladder
K-ras en $31 \%$ en los CVB, sin embargo, no encontraron mutaciones en las displasias o adenomas $^{32}$. Otros autores, han comunicado una baja frecuencia de mutaciones en el gen K-ras en el carcinoma de la vesícula biliar sin unión anómala pancreático-biliar y $25 \%$ en adenomas vesiculares sugiriendo que estas lesiones no serían precursoras del carcinoma invasor de la vesícula biliar8.

En conclusión podemos señalar que la mutación del codón 12 del gen K-ras está presente en al menos el 30\% de nuestros cánceres avanzados de la vesícula biliar, lo que representa un importante avance en el entendimiento de esta neoplasia en nuestra población. El estudio de lesiones metaplásicas, displasia y carcinomas incipientes permitirá establecer con mayor precisión el momento de aparición de esta mutación y su participación en la evolución carcinogenética de la vesícula biliar. carcinoma. J Hepatobiliary Pancreat Surg 1999; 6: 237-44.

8. Wistuba II, Miquel JF, Gazdar AF, Albores-Saavedra J. Gallbladder adenomas have molecular abnormalities different from those present in gallbladder carcinomas. Hum Pathol 1999; 30: 21-5.

9. Lazcano-Ponce EC, Miquel JF, Muñoz N, Herrero R, FerRecio C, Wistuba II et al. Epidemiology and molecular pathology of gallbladder cancer. CA Cancer J Clin 2001; 51: 349-64.

10. RASHID A. Cellular and molecular biology of biliary tract. Surg Oncol Clin N Am 2002; 11: 9951009.

11. Masuhara S, Kasuya $K$, AoK T, Yoshimatsu A, Tsuchida A, Koyanagi Y. Relation between K-ras codon 12 mutation and p53 protein overexpression in gallbladder cancer and biliary ductal epithelia in patients with pancreaticobiliary maljunction. J Hepatobiliary Pancreat Surg 2000; 7: 198-205.

12. Rashid A, Gao YT, Bhakta S, Shen MC, Wang BS, Deng J ET AL. Beta-catenin mutations in biliary tract cancers: a population based study in China. Cancer Res 2001; 61: 3406-9. 
13. Chang HJ, Jee CD, Kм WH. Mutation and altered expression of beta-catenin during gallbladder carcinogenesis. Am J Surg Pathol 2002; 26: 75866.

14. Kim YW, Huh SH, Park YK, Yoon TY, Lee SM, Hong SH. Expression of the c-erb-B2 and p53 protein in gallbladder carcinomas. Oncol Rep 2001; 8: 1127-32.

15. Wistuba II, Gazdar AF, Roa I, Albores-Saavedra J. p53 protein overexpression in gallbladder carcinoma and its precursor lesions: an immunohistochemical study. Hum Pathol 1996; 27: 360-5.

16. Km YT, Km J, Jang YH, Lee WJ, Ryu JK, Park YK et AL. Genetic alterations in gallbladder adenoma, dysplasia and carcinoma. Cancer Lett 2001; 169: 59-68.

17. Shi YZ, Hui AM, L $\mathrm{X}$, Takayama T, Makuuchi $\mathrm{M}$. Overexpression of retinoblastoma protein predicts decreased survival and correlates with loss of p16INK4 protein in gallbladder carcinomas. Clin Cancer Res 2000; 6: 4096-100.

18. Yoshida T, Sugai T, Habano W, Nakamura S, Uesugi $\mathrm{N}$, Funato O ET aL. Microsatellite instability in gallbladder carcinoma: two independent genetic pathways of gallbladder carcinogenesis. J Gastroenterol 2000; 35: 768-74.

19. Itoi $T$, Watanabe $H$, Ajioka $Y$, Oоhashi $Y$, Takel $K$, NishiKURA K ET AL. APC, K-ras codon 12 mutations and p53 gene expression in carcinoma and adenoma of the gallbladder suggest two genetic pathways in gallbladder carcinogenesis. Pathol Int 1996; 46: 333-40.

20. Hidaka E, Yanagisawa A, Sakai Y, SeK M, Ktagawa T, Setoguchi T ET AL. Losses of heterozygosity on chromosomes $17 p$ and 9p/18q may play important roles in early and advanced phases of gallbladder carcinogenesis. J Cancer Res Clin Oncol 1999; 125: 439-43.

21. Watanabe $H$, Date $K$, Itoi $T$, Matsubayashi $H$, Yokoyama N, Yamano M et aL. Histological and genetic changes in malignant transformation of gallbladder adenoma. Ann Oncol 1999; 10: 136-9.

22. Yanagisawa N, Mikami T, Saegusa M, Okayasu I. More frequent beta-catenin exon 3 mutations in gallbladder adenomas than in carcinomas indicate different lineages. Cancer Res 2001; 61: 19-22.

23. Sasatomi E, Tokunaga O, MryazaK K. Precancerous conditions of gallbladder carcinoma: overview of histopathologic characteristics and molecular ge- netic findings. J Hepatobiliary Pancreat Surg 2000; 7: 556-67.

24. Hruban RH, Iacobuzio-Donahue C, Wilentz RE, Goggins M, KeRN SE. Molecular pathology of pancreatic cancer. Cancer J 2001; 7: 251-8.

25. Aunobie B, Sanches R, Didier E, Bignon YJ. Major oncogenes and tumor suppressor genes involved cancer (review). Int J Oncol 2000; 16: 567-76.

26. Eш⿱ CA, Ciark G. The importance of being K-Ras. Cell Signal 2000; 12: 425-34.

27. Feng Z, Hu W, Chen JX, Pao A, Li H, Rom W et al. Preferential DNA damage and poor repair determine ras hotspot in human cancer. J Natl Cancer Inst 2002; 94: 1527-36.

28. Keley MJ, Lttman SJ. Etiology of the mutational spectrum of ras genes in. J Natl Cancer Inst 2002; 94: 1516-7.

29. Almoguera C, Shibata D, Forrester K, Martin J, Arnheim N, Perucho M. Most human carcinomas of the exocrine pancreas contain mutant c-K-ras genes. Cell 1988; 53: 549-54.

30. Ajik T, Fujimori T, Onoyama H, Yamamoto M, Ktazana S, MAEDA S ET AL. K-ras gene mutation in gallbladder cancinomas and dysplasia. Gut 1996; 38: 426-9.

31. Rashid A, Uek T, Gao YT, Houlhan PS, Walaace C, WANG BS ET AL. K-ras mutation, p53 overexpression, and microsatellite instability in biliary tract cancers: a population-based study in China. Clin Cancer Res 2002; 8: 3156-63.

32. Km SW, Her KH, Jang JY, Km WH, Km YT, Park YH. K-ras oncogene mutation in cancer and precancerous lesions of the gallbladder. J Surg Oncol 2000; 75: 246-51.

33. Hanada $K$, Iтoh $M$, Fuji $K$, Tsuchida A, Ooishi $H$, KaJIYAMA G. K-ras and p53 mutations in stage I gallbladder carcinoma with an junction of the pancreaticobiliary duct. Cancer 1996; 77: 452-8.

34. Imai M, Hoshi $T$, Ogawa K. K-ras codon 12 mutations in biliary tract tumors detected by polymerase chain reaction denaturing gradient gel electrophoresis. Cancer 1994; 73: 2727-33.

35. Hanada K, Tsuchida A, Imao T, Eguchi N, SasaK T, MorinaKa K ET AL. Gene mutations of K-ras in gallbladder mucosae and gallbladder with an anomalous junction of the pancreaticobiliary duct. Am J Gastroenterol 1999; 94: 1638-42.

36. RASHID A. Cellular and molecular biology of biliary tract cancers. Surg Oncol Clin N Am 2002; 11: 995-1009. 
37. Minamoto T, Mai M, Ronai Z. K-ras mutation: early detection in molecular of colorectal, pancreas and lung cancers a review. Cancer Detect Prev 2000; 24: 1-12.

38. Wistuba II, Albores-Saavedra J. Genetic abnormalities involved in the pathogenesis of gallbladder carcinoma. J Hepatobiliary Pancreat Surg 1999; 6: $237-44$
39. Nakayama K, Konno M, Kanzak A, Morikawa T, Mirashita H, FujIoKa T et al. Allelotype analysis of gallbladder carcinoma associated with anomalous junction of pancreaticobiliary duct. Cancer Lett 2001; 166: 135-41.

40. AjIKI T, Fujimori T, Onoyama $\mathrm{H}$, Yamamoto $\mathrm{M}$, KitaZawa S, Maeda S, Saito H. K-ras gene mutation in gall bladder carcinomas and dysplasia. Gut. 1996, 38: 426-9. 